\title{
MMO: Multiply-Minus-One Rule for Detecting \& Ranking Positive and Negative Opinion
}

\author{
Sheikh Muhammad Saqib \\ Institute of Computing and information Technology \\ Gomal University \\ Dera Ismail Khan, PAKISTAN
}

\author{
Fazal Masud Kundi \\ Institute of Computing and information Technology \\ Gomal University \\ Dera Ismail Khan, PAKISTAN
}

\begin{abstract}
Hit and hot issue about reviews of any product is sentiment classification. Not only manufacturing company of the reviewed product takes decision about its quality, but the customers' purchase of the product is also based on the reviews. Instead of reading all the reviews one by one, different works have been done to classify them as negative or positive with preprocessing. Suppose from 1000 reviews, there are $\mathbf{3 0 0}$ negative and 700 are positive. As a whole it is positive. Company and customer may not be satisfied with this sentiment orientation. For companies, negative reviews should be separated with respect to different aspects and features, so companies can enhance the features of the product. There is also a lot of work on aspect extraction, and then aspect based sentiment analysis. While on the other hand, users want the most positive reviews and the most negative reviews, then they can decide purchasing a certain product. To consider the issue from users' perspective, authors suggest a method Multiply-Minus-One (MMO) which can evaluate each review and find scores based on positive, negative, intensifiers and negation words using WordNet Dictionary. Experiments on 4 types of datasets of product reviews show that this method can achieve $86 \%, 83 \%, 83 \%$ and $85 \%$ precision performance.
\end{abstract}

Keywords-Sentiment Classification; Preprocessing; Text Mining; Sentiment Orientation

\section{INTRODUCTION}

Positive or negative sentence is classified as opinion. With a single glance, anybody can understand either sentence is positive or negative. But automatic detection of sentence polarity requires some rules. Detection of polarity of sentence is also known as sentiment analysis. For sentiment analysis, subjectivity is very important [1][2]. Subjective sentences are user's opinion while objective sentence has no opinion. Different types of work has different accuracies i,e, final classification accuracies on reviews from various domains range from $84 \%$ for automobile reviews to $66 \%$ for movie reviews [2]. Adjective-noun pair's subjectivity found improved performance in sentiment classification [3]. This subjectivity can be done on document level [4], or sentence level [5]. Using any level, there is opinion related to some entity. Entity and aspect can be extracted using target relations, supervised learning or frequent noun [6][7][8][9]. After the extraction of aspect, sentiment analysis can be done on particular features [10][11]. Work of a supervised learning algorithm determines aspects and then sentiment classification shows the accuracies of $67.37 \%$ and $67.07 \%$ for the restaurants and laptops reviews, respectively [12]. In all above work used text is said as data.
Incomplete, noisy, and inconsistent data requires preprocessing. Noisy data means incorrect attribute values, errors in data transmission, duplicate data etc. Preprocessing process includes [13] irrelevant opinion (non-English) are removed from the data set, duplicate of any opinion are deleted from the data set, stop words, numeric expressions and punctuations are removed, repeated spaces are replaced with single space character, characters repeated 2 or more times in any word are replaced with one or two occurrences for spelling correction if possible, words with all capital letters are identified used for expressing powerful emotions, all tokens starting with "http://", "https://", "http:", "http", or "www." are replaced with <URL>, negations "don't", "didn't" etc. are replaced with "do-not", "did-not". For tokenizing word_tokenize \& tagging, word_tokenize \& pos_tag will be used respectively. And large lexical database of English is implemented in WordNet. It consists of nouns, verbs, adjectives and adverbs are grouped into sets of cognitive synonyms known as synsets. Each synset express separate concept and sentiment scores. In proposed work scores will be taken from WordNet [15].

If there is single line review, then after preprocessing, directly take its polarity with some preprocessing. Now a days anybody want to purchase any product, then search can be made on online search engine. So there is no need to extract entity, because user direct take a jump on review page of required entity. User can purchase the product if as whole product reviews are positive. Aspect based classification is necessary for manufacturing, they can enhance less quality aspect. But end user need the reviews as whole ranked positive or ranked negative reviews.

A rule in which a positive word was given the sentiment score of +1 and a negative word was given the sentiment score of -1 [17], but there is no comparisons between the negative words and positive words, at the end decision (negative or positive) can be made on the basis of overall positive and negative score.

Sentiment shifter [18] also known as negation. Here in this rule a sentence with positive word followed by 'not' will get -1 score i.e. not good [-1]. In this polarity of a review can be found but ranking cannot be obtained. Suppose two sentences "This is bad" and "This is not good" here "not good [-1] = bad [-1]", both sentence has same scores and negative sentences but 1 st one is less negative with respect to 2 nd. 
The algorithm [18] sums up the sentiment scores of the terms in the review considering negations and intensifiers, here positive score of a word is taken as 1 and negative score as -2 . Whole algorithm is working well to handle negation and intensifier. But in case of two negative sentences, both can have same score i.e. this is very bad or this is very wicked. In proposed methodology author will determined 2 nd sentence is more negative then 1 st one.

Keeping this consideration, proposed work planned a method Multiply-Minus-One (MMO) which can determine the polarity of a review. In product reviews it is observed that user express their experiences with product using positive words, negative words, intensifiers and negations. Suppose there are two reviews, i.e. "this is good mobile", "this is not bad mobile", here both are positive but first one is more positive than second one. User says first sentence when they $100 \%$ satisfied with the product and says second sentence when they satisfied up to some extent. Here author proposed a strategy to determine eight rules based on opinionated words, negation and intensifiers. Experimental work on reviews of Hotel, Samsung J7, Lumia-520 and QX25, results have showed precision of $86 \%, 83 \%, 83 \%$ and $85 \%$ respectively.

\section{Multiply-MinUs-ONE (MMO) FOR REVIEW CLASSIFICATION AND RANKING}

As main focus of proposed work is to handle intensifier and negation with opinionated words, so during preprocessing there is no need to remove intensifier and negation, if they exist in stop words list from get_stop_words of NLTK. Here author has manually created a intensifier list (i.e. I: very, more, lot, extra.. etc) and negation list (i.e. N: not, never, none, nobody, nowhere, neither,... etc).

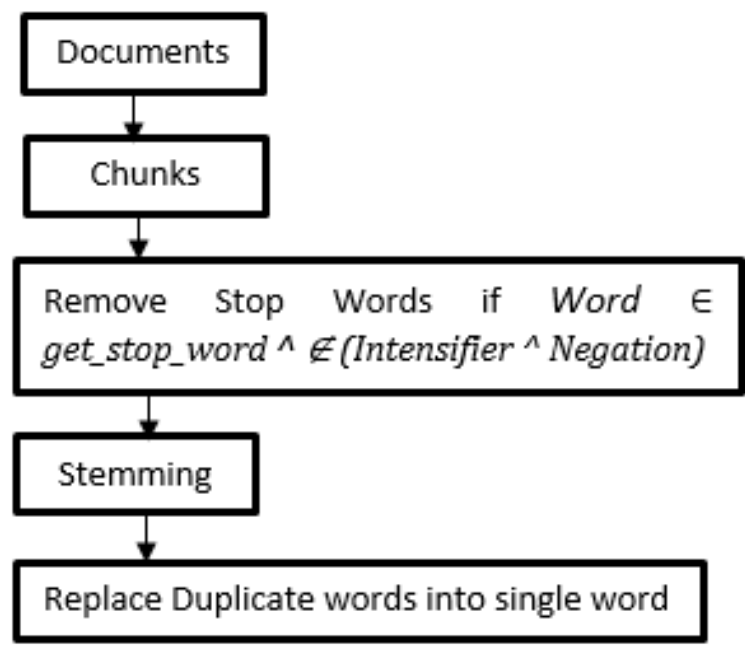

Fig. 1. Preprocessing work

After preprocessing each review consists of chunks and take each chunk with 4 tuples i.e. (W,T,PS,NS). Where w is any subjective word i.e. Adjective (JJ), negation (RB), Intensifier (RB) or even noun (NN). $\mathrm{T}$ is Tag and PS positive score \& NS negative score. Now chunks of a review will be passed through five steps if word meets the objective of each steps.

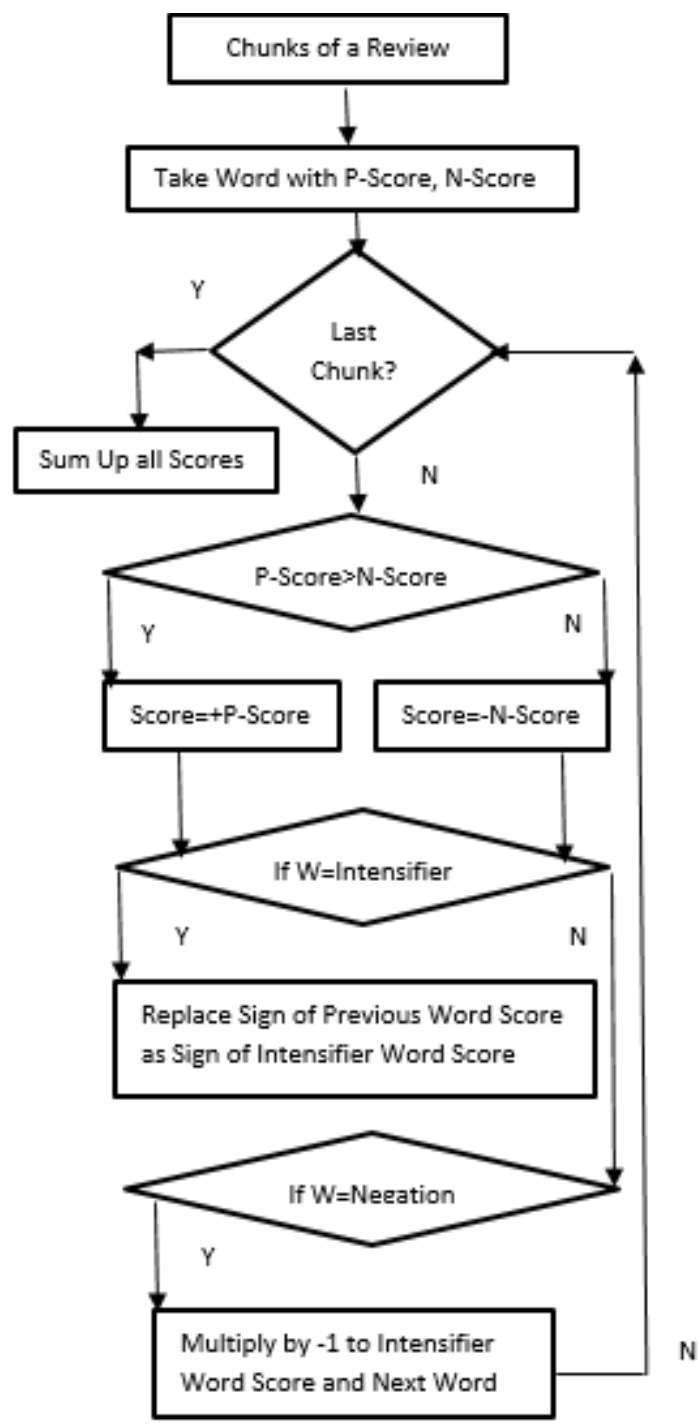

Fig. 2. Flow Chart of MMO

After exploring the whole work of proposed framework, it is concluded that whole work consists of five steps given in following table.

TABLE I. STEPS AFter PRE-PROCESSING

\begin{tabular}{|l|l|l|}
\hline Steps & Objective & Action \\
\hline Step-1 & Chunks & Words with Scores and Tag \\
\hline Step-2 & $\begin{array}{l}\text { Capture } \\
\text { Score }\end{array}$ & $\begin{array}{l}\text { Take Greater Score from -ve and } \\
\text { +ve Scores }\end{array}$ \\
\hline Step-3 & $\begin{array}{l}\text { Intensifier } \\
\text { Handling }\end{array}$ & $\begin{array}{l}\text { Replace sign of Intensifier as next } \\
\text { word (Adjective) }\end{array}$ \\
\hline Step-4 & $\begin{array}{l}\text { Negation } \\
\text { Handling }\end{array}$ & $\begin{array}{l}\text { Replace sign of Intensifier and next } \\
\text { word (Adjective) as negation }\end{array}$ \\
\hline Step-5 & Summation & Sum of All Scores from above Row \\
\hline
\end{tabular}

Positive words can express positive and negative opinion and vice versa. Keeping this thing in mind we have derived eight types of opinions, which will be handled in proposed model.

These steps will work on eight types of opinion. Here we will take simple examples to understand the concepts of 
proposed rules. Each examples consist of five steps as mentioned in Table-1.

\section{A. Positive Opinion with Positive Words}

It is clear that an opinion which contains positive word will depict positive opinion. We can extract polarity of a word using WordNet. If positive score is greater than negative score then then take positive score with +ve sign. Rule for this type of opinion is given below.

$$
(\mathrm{PW}, \mathrm{PS}, \mathrm{JJ})=>\text { +PS ----------------------->Rule-1 }
$$

Where PW is a positive word, PS is its positive score and JJ is adjective.

TABLE II. EXECUTED STEPS FOR RULE-1

\begin{tabular}{|l|l|}
\hline Text-1 \\
\hline Text & This is good mobile \\
\hline Step-1 & ['good', 'JJ', 0.5, 0.0] \\
\hline Step-2 & {$[$ 'good', 'JJ',+ 0.5] } \\
\hline Step-3 & ['good', 'JJ', 0.5] \\
\hline Step-4 & {$[$ 'good', 'JJ', 0.5] } \\
\hline Step-5 & $\mathbf{+ 0 . 5}$ \\
\hline
\end{tabular}

In above example there is no intensifier and negation, so only step-1, step-2 and step-5 will be performed. Score of this opinion is +0.5 , means opinion is positive.

\section{B. Positive Opinion with Positive Words and Intensifiers}

As Intensifier with positive words increases the intensity of positive opinion, so we also consider the intensifier score with positive word.

$(\mathrm{I}, \mathrm{ISC}, \mathrm{RB})^{\wedge}(\mathrm{PW}, \mathrm{PS}, \mathrm{JJ})=>(+\mathrm{PS})+(+\mathrm{ISC})$ $------>$ Rule-2

Where I is intensifier, ISC is its score, PW is positive word, PS is its positive score. Place +ve sign with ISC as sign of PS.

TABLE III. EXECUTED STEPS For RULE-2

\begin{tabular}{|l|l|}
\hline Text-2 & \multicolumn{1}{|l|}{} \\
\hline Text & This is very good mobile \\
\hline Step-1 & ['very', 'RB', 0.25, 0.25, 'good', 'JJ', 0.5, 0.0] \\
\hline Step-2 & ['very', 'RB', 0.25, 'good', 'JJ',+ 0.5] \\
\hline Step-3 & ['very', 'RB', +0.25, 'good', 'JJ', +0.5] \\
\hline Step-4 & ['very', 'RB', 0.25, 'good', 'JJ', 0.5] \\
\hline Step-5 & $\mathbf{+ 0 . 7 5}$ \\
\hline
\end{tabular}

As there is intensifier, so step-3 will be performed to change the sign of RB $(+0.25)$ as $\mathrm{JJ}(+0.5)$.

Hence Step-1, Step-2, Step-3 and Step-5 will be performed to get final score. Here final score is +0.75 means it is more positive opinion then without intensifier.

\section{Negative Opinion with Negation and Positive Words}

As if word is positive then its negation make its concept as negative, means multiply the positive score of $\mathrm{JJ}$ with -1 if previous word is negation.

$(\mathrm{N},-\mathrm{NSC}, \mathrm{RB})^{\wedge}(\mathrm{PW}, \mathrm{PS}, \mathrm{JJ})=>(-\mathrm{NSC})+(\mathrm{PS} *(-1.0))-----$ $->$ Rule-3
Where $\mathrm{N}$ is negation, -NSC is negative score of negation, PW is positive word and PS is its positive score. In resultant side, there is PS * $(-1)$ because previous word is negation.

TABLE IV. EXECUTED STEPS FOR RULE-3

\begin{tabular}{|l|l|}
\hline \multicolumn{2}{|l|}{ Text-3 } \\
\hline Text & This is not good mobile \\
\hline Step-1 & ['not', 'RB', 0.0, 0.625, 'good', 'JJ', 0.5, 0.0] \\
\hline Step-2 & ['not', 'RB', '-0.625', 'good', 'JJ',+ 0.5] \\
\hline Step-3 & ['not', 'RB', '-0.625', 'good', 'JJ', 0.5] \\
\hline Step-4 & {$[$ 'not', 'RB', '-0.625', 'good', 'JJ', -0.5] } \\
\hline Step-5 & $-\mathbf{1 . 1 2 5}$ \\
\hline
\end{tabular}

As there is negation, so step-4will be performed to multiply positive score of $\mathrm{JJ}(0.5)$ with -1.0 .

Hence Step-1, Step-2, Step-4 and Step-5 will be performed to get final score. Here final score is -1.125 means it is negative opinion.

\section{Negative Opinion with Negation, Positive Words and Intensifiers}

As positive word with negation shows that the opinion is negative, if there is intensifier then opinion will be more negative. So to handle intensifier, apply Rule-2 and for negation apply Rule-3. Resultant rule will be as:

$$
\begin{aligned}
& (\mathrm{N},-\mathrm{NSC}, \mathrm{RB}) \wedge(\mathrm{I}, \mathrm{ISC}, \mathrm{RB}) \wedge(\mathrm{PW}, \mathrm{PS}, \mathrm{JJ}) \\
& =>(\mathrm{N},-\mathrm{NSC}, \mathrm{RB}) \wedge(\mathrm{I},+\mathrm{ISC}, \mathrm{RB}) \wedge(\mathrm{PW},+\mathrm{PS}, \mathrm{JJ}) \\
& =>(-\mathrm{NSC})+(\mathrm{ISC} *(-1.0))+(\mathrm{PS} *(-1.0))------
\end{aligned}
$$

First of all replace the sign of Intensifier score (+ISC) as positive word score (+PS), then multiply both of them with -1 , because there is negation before them.

\section{TABLE V. EXECUTED STEPS FOR RULE-4}

\begin{tabular}{|l|l|}
\hline Text-4 \\
\hline Text & This is not very good mobile \\
\hline Step-1 & $\begin{array}{l}\text { ['not', 'RB', 0.0, 0.625, 'very', 'RB', } \\
\mathbf{0 . 2 5 ,} \mathbf{0 . 2 5}, \text { 'good', 'JJ', 0.5, 0.0] }\end{array}$ \\
\hline Step-2 & $\begin{array}{l}\text { ['not', 'RB', '-0.625', 'very', 'RB',+ } \\
\text { 0.25, 'good', 'JJ',+ 0.5] }\end{array}$ \\
\hline Step-3 & $\begin{array}{l}\text { ['not', 'RB', '-0.625', 'very', 'RB',+ } \\
\text { 0.25, 'good', 'JJ', +0.5] }\end{array}$ \\
\hline Step-4 & $\begin{array}{l}\text { ['not', 'RB', '-0.625', 'very', 'RB', -0.25, } \\
\text { 'good', 'JJ', -0.5] }\end{array}$ \\
\hline Step-5 & $-\mathbf{- 1 . 3 7 5}$ \\
\hline
\end{tabular}

As there is intensifier and negation, so Step-3 and Step-4 both will be considered. For intensifier handling, replace the sign of intensifier $(+0.25)$ as adjective $(+0.5)$ and then to handle negation multiply them with -1.0 i.e intensifier $(+0.25 *$ $(-1.0))$ as adjective $(+0.5 *(-1.0))$. So final score will be 1.375 means negative opinion.

\section{E. Negative Opinion with Negative Words}

If an opinion contains just negative word, then take its negative score with -ve sign. Its rule will be generated as:

$(\mathrm{NW}, \mathrm{NS}, \mathrm{JJ})=>$-NS --------------------->Rule-5 
Where NW is a negative word, NS is negative score, resultant value with -ve sign shows that opinion is negative.

TABLE VI. EXECUTED STEPS FOR RULE-5

\begin{tabular}{|l|l|}
\hline Text-5 & \\
\hline Text & This is bad mobile \\
\hline Step-1 & ['bad', 'JJ', $\mathbf{0 . 0 , 0 . 8 7 5}]$ \\
\hline Step-2 & ['bad', 'JJ', '-0.875'] \\
\hline Step-3 & ['bad', 'JJ', '-0.875'] \\
\hline Step-4 & ['bad', 'JJ', '-0.875'] \\
\hline Step-5 & $\mathbf{- 0 . 8 7 5}$ \\
\hline
\end{tabular}

In above example there is no intensifier and negation, so only step-1, step-2 and step-5 will be performed. Score of this opinion is -0.875 , means opinion is negative.

\section{F. Negative Opinion with Negative Words and Intensifiers}

As intensifier with negative words also increases the intensity of negative opinion, so we also consider the intensifier score with negative word. As mostly intensifier are positive, so there is need to change its score as negative using following rule:

$$
(\mathrm{I}, \mathrm{ISC}, \mathrm{RB}) \wedge(\mathrm{NW},-\mathrm{NS}, \mathrm{JJ})=>(\mathrm{ISC} *(-1.0))+(-\mathrm{NS})------
$$

Where I is intensifier, ISC is its score, NW is positive word, -NS is its negative score. Place -ve sign with ISC as sign of NS.

TABLE VII. EXECUTED STEPS FOR RULE-6

\begin{tabular}{|l|l|}
\hline Text-6 & \\
\hline Text & This is very bad mobile \\
\hline Step-1 & $\begin{array}{l}\text { ['very', 'RB', 0.25, 0.25, 'bad', 'JJ', 0.0, } \\
\text { 0.875] }\end{array}$ \\
\hline Step-2 & ['very', 'RB', 0.25, 'bad', 'JJ', '-0.875'] \\
\hline Step-3 & ['very', 'RB', -0.25, 'bad', 'JJ', '-0.875'] \\
\hline Step-4 & ['very', 'RB', -0.25, 'bad', 'JJ', '-0.875'] \\
\hline Step-5 & $\mathbf{- 1 . 1 2 5}$ \\
\hline
\end{tabular}

As there is intensifier, so, step-3 will be performed to change the sign of RB (-0.25) as JJ (-0.875). Hence Step-1, Step-2, Step-3 and Step-5 will be performed to get final score. Here final score is -1.125 means it is more negative opinion then without intensifier.

\section{G. Positive Opinion with Negation and Negative Words}

As if word is negative then its negation make its concept as positive, means multiply the multiply score of JJ with -1 if previous word is negation.

$$
(\mathrm{N},-\mathrm{NSC}, \mathrm{RB})^{\wedge}(\mathrm{NW},-\mathrm{NS}, \mathrm{JJ})=>(-\mathrm{NSC})+(-\mathrm{NS} *(-1.0))-
$$
$->$ Rule-7

Where $\mathrm{N}$ is negation, -NSC is negative score of negation, $\mathrm{NW}$ is negative word and -NS is its negative score. In resultant side, there is $(-\mathrm{NS}) *(-1)$ because previous word is negation.
TABLE VIII. EXECUTED STEPS FOR RULE-7

\begin{tabular}{|l|l|}
\hline \multicolumn{2}{|l|}{ Text-7 } \\
\hline Text & This is not bad mobile \\
\hline Step-1 & ['not', 'RB', 0.0, 0.625, 'bad', 'JJ', 0.0, 0.875] \\
\hline Step-2 & ['not', 'RB', '-0.625', 'bad', 'JJ', '-0.875'] \\
\hline Step-3 & {$[$ 'not', 'RB', '-0.625', 'bad', 'JJ', '-0.875'] } \\
\hline Step-4 & {$[$ ['not', 'RB', '-0.625', 'bad', 'JJ', 0.875] } \\
\hline Step-5 & $\mathbf{0 . 2 5}$ \\
\hline
\end{tabular}

As there is negation, so, step-4 will be performed to multiply negative score of JJ (-0.625) with -1.0.

Hence Step-1, Step-2, Step-4 and Step-5 will be performed to get final score. Here final score is 0.25 means it is positive opinion.

\section{H. Positive Opinion with Negation, Negative Words and Intensifiers}

As negative word with negation shows that the opinion is positive, if there is intensifier then opinion will be more positive. So to handle intensifier, apply Rule-6 and for negation apply Rule-7. Resultant rule will be as:

$$
\begin{aligned}
& (\mathrm{N}, \mathrm{NSC}, \mathrm{RB}) \wedge(\mathrm{I}, \mathrm{ISC}, \mathrm{RB})^{\wedge}(\mathrm{NW},-\mathrm{NS}, \mathrm{JJ}) \\
& =>(\mathrm{N},-\mathrm{NSC}, \mathrm{RB})^{\wedge}(\mathrm{I},-\mathrm{ISC}, \mathrm{RB}) \wedge(\mathrm{PW},-\mathrm{NS}, \mathrm{JJ}) \\
& \Rightarrow>(-\mathrm{NSC})+(\mathrm{ISC} *(-1.0))+(-\mathrm{NS} *(-1.0))---
\end{aligned}
$$

\begin{tabular}{|c|c|}
\hline \multicolumn{2}{|l|}{ Text-8 } \\
\hline Text & This is not very bad mobile \\
\hline Step-1 & $\begin{array}{l}\text { ['not', 'RB', 0.0, 0.625, 'very', 'RB', } \\
0.25,0.25, \text { 'bad', 'JJ', 0.0, 0.875] }\end{array}$ \\
\hline Step-2 & $\begin{array}{l}\text { ['not', 'RB', '-0.625', 'very', 'RB', } \\
\text { 0.25, 'bad', 'JJ', '-0.875'] }\end{array}$ \\
\hline Step-3 & $\begin{array}{l}\text { ['not', 'RB', '-0.625', 'very', 'RB', - } \\
0.25, \text { 'bad', 'JJ', '-0.875'] }\end{array}$ \\
\hline Step-4 & $\begin{array}{l}\text { ['not', 'RB', '-0.625', 'very', 'RB', } \\
\text { 0.25, 'bad', 'JJ', 0.875] }\end{array}$ \\
\hline Step-5 & 0.5 \\
\hline
\end{tabular}$$
\text { ----> Rule-8 }
$$

First of all replace the sign of Intensifier score (-ISC) as negative word score (-NS), then multiply both of them with -1 , because there is negation before them.

TABLE IX. EXECUTED STEPS FOR RULE-8

As there is intensifier and negation, so Step-3 and Step-4 both will be considered. For intensifier handling, replace the sign of intensifier $(-0.25)$ as adjective $(-0.5)$ and then to handle negation multiply them with -1.0 i.e intensifier $(-0.25 *(-1.0))$ as adjective $(-0.5 *(-1.0))$. So, final score will be 0.5 means positive opinion.

\section{ALGORITHM OF MMO}

After merging the above eight rules in five steps (given in Table-1), algorithm of proposed work is plotted in Table-10. 
TABLE X. ALGORITHM OF PROPOSED WORK

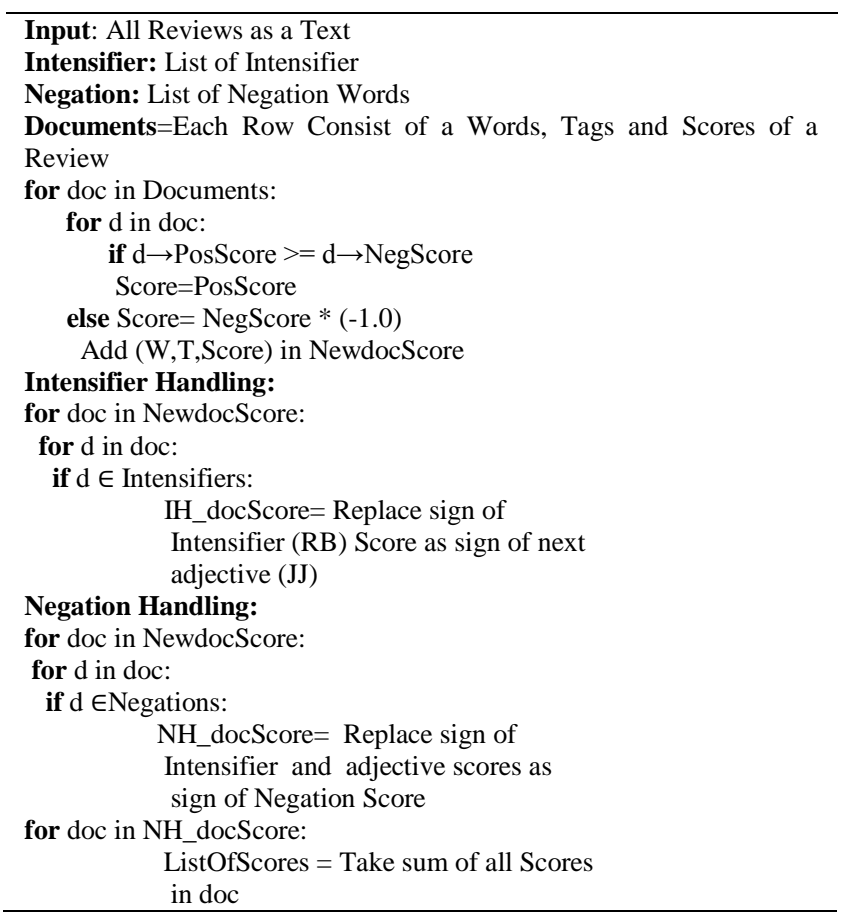

\section{CONCLUSION AND RESULTS}

User of a product only requires to know whether the product on the whole is negative or positive, while the manufacturing company would require a sorted list of reviews with respect to negative to positive impact i.e. most negative review should be placed at the top of the list, then next negative review and so on. So, on the basis that sorted list, they can enhance the quality of the features discussed in the negative reviews, according to their respective intensity. The purpose of the proposed framework is to sort all the reviews with respect to its sensitivity. Following table is representing the reviews from negative to positive score, calculating from eight said rules.

TABLE XI. SORTED REVIEWS BASED ON SENTIMENT SCORES

\begin{tabular}{|l|l|l|}
\hline Text Tag & Text & Scores \\
\hline T4 & This is not very good mobile & -1.375 \\
\hline T3 & This is not good mobile & -1.125 \\
\hline T6 & This is very bad mobile & -1.125 \\
\hline T5 & This is bad mobile & -0.875 \\
\hline T7 & This is not bad mobile & 0.25 \\
\hline T1 & This is good mobile & 0.5 \\
\hline T8 & This is not very bad mobile & 0.5 \\
\hline T2 & This is very good mobile & 0.75 \\
\hline
\end{tabular}

Fig-3 is showing that T4 is high negative and T2 is high positive comment.

Proposed work has been applied on reviews of Hotel, Samsung-J7, Lumia-520 and QX25 products. Each product review consists of different types of intensifiers and negations. Positive predicted value is known as precision and recall means

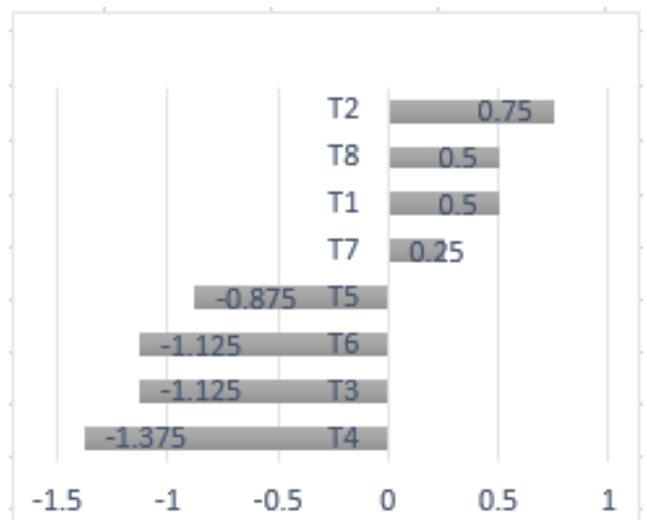

Fig. 3. Ranked Reviews

sensitivity i.e. large recall value means a few positive cases misclassified as a negative [16]. Both can be calculated through following formulas:

Precision $=\mathrm{TP} /(\mathrm{TP}+\mathrm{FP}), \quad$ Recall $=\mathrm{TP} /(\mathrm{TP}+\mathrm{FN})$

Where TP is True Positive (Number of positive reviews classified correctly), FN is False Negative (Number of positive reviews classified incorrectly as a negative), $\mathrm{TN}$ is True Negative (Number of negative reviews classified correctly) and FP is False Positive (Number of negative reviews classified incorrectly as a positive).

TABLE XII. RESULTS BASED ON PROPOSED WORK

\begin{tabular}{|l|l|l|l|l|l|l|}
\hline Category & TP & FN & TN & FP & $\begin{array}{l}\text { Precisio } \\
\text { n }\end{array}$ & $\begin{array}{l}\text { Recal } \\
\text { l }\end{array}$ \\
\hline Hotel & $89 \%$ & $11 \%$ & $16 \%$ & $14 \%$ & $86 \%$ & $89 \%$ \\
\hline J7 & $\begin{array}{l}71.43 \\
\%\end{array}$ & $\begin{array}{l}28.57 \\
\%\end{array}$ & $\begin{array}{l}42.86 \\
\%\end{array}$ & $\begin{array}{l}14.29 \\
\%\end{array}$ & $83 \%$ & $71 \%$ \\
\hline Lumia-520 & $50 \%$ & $10 \%$ & $40 \%$ & $10 \%$ & $83 \%$ & $83 \%$ \\
\hline QX25 & $\begin{array}{l}54.55 \\
\%\end{array}$ & $\begin{array}{l}18.18 \\
\%\end{array}$ & $\begin{array}{l}27.27 \\
\%\end{array}$ & $9.09 \%$ & $85 \%$ & $75 \%$ \\
\hline
\end{tabular}

Here we have mentioned some reviews of $\mathbf{J} 7$, with scores in sorted form, so user can read most sensitive review in advance.

TABLE XIII. SORTED REVIEWS BASED ON SCORE

\begin{tabular}{|l|l|}
\hline Reviews & Scores \\
\hline $\begin{array}{l}\text { worst mobile, sensitive touch display+network } \\
\text { problems bad in this mobile }\end{array}$ & -2.5 \\
\hline $\begin{array}{l}\text { my J7 and my friends J7 mobile data network is } \\
\text { not working properly sometimes it seems to be } \\
\text { unavailable. }\end{array}$ & -1.875 \\
\hline $\begin{array}{l}\text { worst performing smartphone till now.......low } \\
\text { ram,low resolution only }\end{array}$ & -1.0 \\
\hline \begin{tabular}{l} 
J7 is the Best Smartphone. Superbbbbb... \\
\hline $\begin{array}{l}\text { I got this phone for about 4 months now..since } \\
\text { then I have not face any logging issue, camera is } \\
\text { good, very smooth, fast charging, for me it is the } \\
\text { best phone at midrense, theres no issue even the } \\
\text { high graphic games. }\end{array}$
\end{tabular} & 0.625 \\
\hline $\begin{array}{l}\text { i never faced any issues during my use of the } \\
\text { phone. also added is some new cloud function, } \\
\text { performance and security updates. }\end{array}$ & 1.0 \\
\hline
\end{tabular}




\section{REFERENCES}

[1] Yu, Hong and Vasileios Hatzivassiloglou. Towards answering opinion questions: Separating facts from opinions and identifying the polarity of opinion sentences. in Proceedings of Conference on Empirical Methods in Natural Language Processing 2003.

[2] Turney, Peter D. Thumbs up or thumbs down?: semantic orientation applied to unsupervised classification of reviews. in Proceedings of Annual Meeting of the Association for Computational Linguistics (ACL-2002).

[3] FelixHill, Anna Korhonen. Concreteness and Subjectivity as Dimensions of Lexical Meaning. Proceedings of the 52nd Annual Meeting of the Association for Computational Linguistics (Short Papers), pages 725-731, Baltimore, Maryland, USA, June 23-25 2014. Association for Computational Linguistic

[4] Pang, Bo, Lillian Lee, and Shivakumar Vaithyanathan. Thumbs up? Sentiment classification using machine learning techniques. in Proceedings of Conference on Empirical Methods in Natural Language Processing (EMNLP-2002).

[5] Wiebe, Janyce, Theresa Wilson, Rebecca F. Bruce, Matthew Bell, and Melanie Martin. Learning subjective language. Computational Linguistics, 2004. 30(3): p. 277-308.

[6] Jingbo Zhu Huizhen Wang, Benjamin K. Tsou, Muhua Zhu. MultiAspect Opinion Polling from Textual Reviews, Proceedings of ACM International Conference on Information and Knowledge Management (CIKM-2009). 2009.

[7] Chong Long, Jie Zhang, Xiaoyan Zhu.A Review Selection Approach for Accurate Feature Rating Estimation. Proceedings of Coling 2010, Poster Volume. 2010.

[8] Lei Zhang, Bing Liu, Aspect and Entity Extraction for Opinion Mining, Data Mining and Knowledge Discovery for Big Data Studies in Big Data Volume 1, 2014, pp 1-40

[9] Zhiyuan Chen, Arjun Mukherjee and Bing Liu, Aspect Extraction with Automated Prior Knowledge Learning, Proceedings of the 52nd Annual
Meeting of the Association for Computational Linguistics, pages 347358, Baltimore, Maryland, USA, June 23-25 2014.

[10] D. K. Kirange1, Ratnadeep R. Deshmukh.ASPECT BASED SENTIMENT ANALYSIS SEMEVAL-2014 TASK 4.Asian Journal of Computer Science And Information Technology 4 : 8 (2014) 72 - 75.

[11] Tomas Brychcin, Michal Konkol, Josef Steinberger. Machine Learning Approach to Aspect-Based Sentiment Analysis. Proceedings of the 8th International Workshop on Semantic Evaluation (SemEval 2014), pages 817-822, Dublin, Ireland, August 23-24, 2014.

[12] Deepak Kumar Gupta,Asif Ekbal. Supervised Machine Learning for Aspect based Sentiment Analysis. Proceedings of the 8th International Workshop on Semantic Evaluation (SemEval 2014), pages 319-323, Dublin, Ireland, August 23-24, 2014.

[13] Fazal Masud Kundi, Aurangzeb Khan, Shakeel Ahmad, Muhammad Zubair Asghar. Web Lexicon-Based Sentiment Analysis in the Social 4(6)238-248, 2014. Journal of Basic and Applied Scientific Research

[14] Dive Into NLTK, Part II: Sentence Tokenize and Word Tokenize. http://textminingonline.com/dive-into-nltk-part-ii-sentence-tokenizeand-word-tokenize

[15] What is WordNet? https://wordnet.princeton.edu/

[16] David L. Olson, Dursun Delen, 2008. PerformanceEvaluation for Predictive Modeling. In: Advanced Data Mining Techniques pp: 137139. Springer-Verlag Berlin Heidelberg.

[17] Kim, Soo-Min and Eduard Hovy. Determining the sentiment of opinions. In Proceedings of Interntional Conference on Computational Linguistics (COLING-2004). 2004.

[18] Polanyi, Livia and Annie Zaenen. Contextual valence shifters. In Proceedings of the AAAI Spring Symposium on Exploring Attitude and Affect in Text. 2004.

[19] Wan, Xiaojun. Using bilingual knowledge and ensemble techniques for unsupervised Chinese sentiment analysis. in Proceedings of Conference on Empirical Methods in Natural Language Processing (EMNLP-2008). 